\title{
Biosynthesis, characterization, and antimicrobial applications of silver nanoparticles
}

\author{
This article was published in the following Dove Press journal: \\ International Journal of Nanomedicine \\ 31 March 2015 \\ Number of times this article has been viewed
}

\author{
Priyanka Singh' \\ Yeon Ju Kim² \\ Hina Singh' \\ Chao Wang ${ }^{2}$ \\ Kyu Hyon Hwang ${ }^{3}$ \\ Mohamed El-Agamy Farh' \\ Deok Chun Yang ${ }^{1,2}$ \\ 'Department of Oriental Medicinal \\ Material and Processing, ${ }^{2} \mathrm{Graduate}$ \\ School of Biotechnology and Ginseng \\ Bank, College of Life Sciences, Kyung \\ Hee University, Yongin, ${ }^{3}$ Gyeonggi-Do \\ Agricultural Research \& Extension \\ Services, Gyeonggi, Republic of Korea
}

Correspondence: Deok Chun Yang/ Yeon Ju Kim

Graduate School of Biotechnology and Ginseng Bank, College of Life Sciences, Kyung Hee University, Yongin, 446-70I, Republic of Korea

$\mathrm{Tel}+823 \mathrm{I} 20 \mathrm{I} 2100$

Fax +82 31 2052688

Email dcyang@khu.ac.kr/

yeonjukim@khu.ac.kr

\begin{abstract}
In the present study, the strain Brevibacterium frigoritolerans DC2 was explored for the efficient and extracellular synthesis of silver nanoparticles. These biosynthesized silver nanoparticles were characterized by ultraviolet-visible spectrophotometry, which detected the formation of silver nanoparticles in the reaction mixture and showed a maximum absorbance at $420 \mathrm{~nm}$. In addition, field emission transmission electron microscopy revealed the spherical shape of the nanoparticles. The dynamic light scattering results indicated the average particle size of the product was $97 \mathrm{~nm}$ with a 0.191 polydispersity index. Furthermore, the product was analyzed by energy dispersive X-ray spectroscopy, X-ray diffraction, and elemental mapping, which displayed the presence of elemental silver in the product. Moreover, on a medical platform, the product was checked against pathogenic microorganisms including Vibrio parahaemolyticus, Salmonella enterica, Bacillus anthracis, Bacillus cereus, Escherichia coli, and Candida albicans. The nanoparticles demonstrated antimicrobial activity against all of these pathogenic microorganisms. Additionally, the silver nanoparticles were evaluated for their combined effects with the commercial antibiotics lincomycin, oleandomycin, vancomycin, novobiocin, penicillin $\mathrm{G}$, and rifampicin against these pathogenic microorganisms. These results indicated that the combination of antibiotics with biosynthesized silver nanoparticles enhanced the antimicrobial effects of antibiotics. Therefore, the current study is a demonstration of an efficient biological synthesis of silver nanoparticles by $B$. frigoritolerans DC2 and its effect on the enhancement of the antmicrobial efficacy of well-known commercial antibiotics.
\end{abstract}

Keywords: Brevibacterium frigoritolerans, biosynthesis, silver nanoparticles, antimicrobial activity, synergistic effect

\section{Introduction}

Currently, the development of a biological methodology for the synthesis of nanoparticles as well as for their characterization and applications on medical and environmental platforms are important aspects of nanotechnology. ${ }^{1,2}$ There is a particularly pressing need for new and efficient methodologies for the synthesis of nanomaterials. Various physical and chemical methods have been reported along with many drawbacks that limit the use of nanoparticles in biological applications; these disadvantages include the use of toxic solvents, the generation of hazardous byproducts, and high energy consumption. The demand for a methodology that would avoid the hazardous byproducts associated with current physicochemical processes leads to the exploration of a biological species capable of synthesizing nanoparticles. ${ }^{3}$ The concept of exploiting biological diversity for the synthesis of nanoparticles promises to be quite simple and economical. In addition, these species have been proved to be free of the limitations associated with chemical and physical synthesis. Furthermore, the use of these organisms represents new product development and may allow for applications of biological resources in various fields. ${ }^{4}$ In the recent past, nanoparticles have been 
synthesized by using various plant extracts, actinomycetes, fungi, and bacteria. ${ }^{5-8}$ The microbial resource is a striking opportunity for nanotechnology, as a number of microorganisms are available and can be explored for this action. ${ }^{9}$

From a medical perspective, the development of resistance mechanisms against antibiotics by pathogenic microorganisms has been a subject of major concern. ${ }^{10}$ These resistance mechanisms, due to several enzymatic and genetic mutations in the pathogens that cause infectious diseases, has encouraged researchers to design new antimicrobial agents against pathogens to control infections. ${ }^{10,11}$ Therefore, there is always a need to develop new alternatives to control pathogenic organisms.

Metal nanoparticles are an effective way to control many pathogenic and antibiotic-resistant microorganisms. ${ }^{12}$ Nanoparticles are applicable in diverse areas such as medicine, diagnostic agents, drug and gene delivery, electronics, cosmetics, coatings, biosensors, imaging, and environmental remediation. Among the many metal nanoparticles, silver nanoparticles have been intensely studied because of the distinct properties of their optical behavior, conductivity, chemical stability, and catalytic activity. ${ }^{13}$ In addition, silver nanoparticles exhibit broad spectrum bactericidal and fungicidal activity. These properties make them suitable for use as a disinfectant in many medical devices and goods. In addition, silver nanoparticles and antibiotic conjugates can be made efficiently and could result in an increase in the efficacy of antibiotics against various pathogenic, antibioticresistant microorganisms. ${ }^{14}$ Because of the applications of silver nanoparticles in the fields of biology, environment, and technology, there is a growing need for the development of a cost-effective method for the biosynthesis of silver nanoparticles.

The present research was carried out to explore a biological method for the synthesis of silver nanoparticles and to apply these nanoparticles in controlling pathogenic microorganisms. Silver nanoparticles were biosynthesized by Brevibacterium frigoritolerans DC2, which was isolated from a fermented food product. These nanoparticles were tested against different pathogenic microorganisms for their antibacterial efficacy both independently and in combination with various conventional antibiotics.

\section{Materials and methods \\ Materials}

All of the media were purchased from MB Cell (Los Angeles, $\mathrm{CA}, \mathrm{USA})$. Analytical grade silver nitrate $\left(\mathrm{AgNO}_{3}\right)$ and cycloheximide were purchased from Sigma-Aldrich Co.
(St Louis, MO, USA). Standard antibiotic discs with standard concentrations for their action against microorganisms were used for the antimicrobial tests. The antibiotics discs that were used are as follows: 1) vancomycin, $30 \mu \mathrm{g} / \mathrm{disc}$; 2) rifampicin, $5 \mu \mathrm{g} / \mathrm{disc}$; 3) oleandomycin, $15 \mu \mathrm{g} / \mathrm{disc}$; 4) penicillin $\mathrm{G}, 10 \mu \mathrm{g} / \mathrm{disc}$; 5) novobiocin, $30 \mu \mathrm{g} / \mathrm{disc}$; and 6) lincomycin, $15 \mu \mathrm{g} / \mathrm{disc}$. All of the discs were purchased from Oxoid Ltd. (Basingstoke, England).

The pathogenic bacterial strains Vibrio parahaemolyticus ATCC 33844, Bacillus anthracis NCTC 10340, Salmonella enterica ATCC 13076, Bacillus cereus ATCC 14579, Escherichia coli ATCC 10798, and Candida albicans KACC 30062 were used. The bacterial strains were cultured on nutrient agar media at $37^{\circ} \mathrm{C}$ and preserved at $-70^{\circ} \mathrm{C}$ in glycerol stock vials for further study. C. albicans was cultured on Sabouraud dextrose agar at $28^{\circ} \mathrm{C}$ and preserved at $-70^{\circ} \mathrm{C}$ in glucose yeast peptone broth glycerol stock vials.

\section{Isolation and molecular identification of bacteria}

Fermented skim yogurt was obtained from Indonesia. The food sample was serially diluted in sterile $0.8 \% \mathrm{NaCl}$ and then spread onto Tryptic soy agar (TSA) media to obtain isolated colonies. To check the metal-tolerating capacity of isolated strains, the strains were further streaked on TSA medium supplemented with $1 \mathrm{mM}$ filter-sterilized $\mathrm{AgNO}_{3}$. The plate was then incubated at room temperature for 48 hours and observed for bacterial growth. The isolated colonies were subcultured and obtained in pure form for further experiments.

Molecular identification of the isolated strain was carried out using a $16 \mathrm{~S}$ ribosomal ribonucleic acid (rRNA) sequencing-based method. The genomic deoxyribonucleic acid (DNA) was extracted by using a commercial genomic DNA extraction kit (Core Bio System, Seoul, Korea). The 16S rRNA gene was amplified from the chromosomal DNA of the isolated strain by using the universal bacterial primer sets $27 \mathrm{~F}, 518 \mathrm{~F}, 800 \mathrm{R}$, and $1512 \mathrm{R} .{ }^{15,16}$ The purified polymerase chain reaction products were sequenced by Genotech (Daejeon, Korea). The nearly complete sequence (1485 base pairs) of the 16S rRNA was compiled by using SeqMan software (version 4.1). The 16S rRNA gene sequences of related taxa were obtained from the GenBank database and EzTaxon-e server. ${ }^{17}$

\section{Biosynthesis of silver nanoparticles}

For biological synthesis of silver nanoparticles, the selected bacterial isolate was inoculated into a $250 \mathrm{~mL}$ Erlenmeyer 
flask containing $100 \mathrm{~mL}$ of sterile Tryptic soy broth. The cultured flasks were incubated in a rotating shaker set at $37^{\circ} \mathrm{C}$ for 120 rotations per minute (rpm) for 24 hours. After the incubation time, the culture was centrifuged at $8,000 \mathrm{rpm}$ for 10 minutes to remove the bacterial pellet. The supernatant was obtained and was mixed with a filtersterilized $\mathrm{AgNO}_{3}$ solution ( $1 \mathrm{mM}$ final concentration) for the extracellular production of silver nanoparticles ${ }^{1,4,7,8}$ Furthermore, the culture supernatant with $1 \mathrm{mM} \mathrm{AgNO}_{3}$ was incubated in an orbital shaker at $200 \mathrm{rpm}$ and $25^{\circ} \mathrm{C}$. The synthesis of silver nanoparticles was monitored by visual inspection for a change in the color of the culture medium. After the completion of the incubation period, the mixture was first centrifuged at 2,000 rpm for 5 minutes to remove any medium components, and then the silver nanoparticles were collected by high speed centrifugation at $16,000 \mathrm{rpm}$ for 20 minutes. The obtained product was washed several times by centrifugation and was redispersed in water to remove the unconverted silver ions and any medium components. Finally, the silver nanoparticles were collected in the form of a pellet and were used for characterization. ${ }^{18}$

\section{Characterization of silver nanoparticles}

To verify the reduction of silver ions, the solution was scanned in the range of $200-800 \mathrm{~nm}$ in a ultraviolet-visible (UV-Vis) spectrophotometer (Ultrospec 2100 Pro; GE Healthcare Bio-Sciences Corp., Piscataway, NJ, USA). The size-distribution profile of silver nanoparticles was studied by using dynamic light scattering with a particle size analyzer (Photal, Otsuka Electronics Co., Osaka, Japan). The hydrodynamic diameters and polydispersity index were analyzed at $25^{\circ} \mathrm{C}$. As a reference dispersive medium, pure water with a refractive index 1.3328 , viscosity 0.8878 and dielectric constant 78.3 was used. The shape, morphology, and elemental distribution of the nanoparticles were analyzed by using field emission transmission electron microscopy (FE-TEM), energy dispersive X-ray spectroscopy (EDX), and elemental mapping with a JEM-2100F (JEOL, Tokyo, Japan) operated at $200 \mathrm{kV}$. The sample was prepared by placing a drop of collected silver nanoparticles on a carbon-coated copper grid and subsequently drying the sample in an oven at $60^{\circ} \mathrm{C}$ before transferring it to the microscope. The X-ray diffraction (XRD) analyses were performed on an X-ray diffractometer, D8 Advance (Bruker, Billerica, MA, USA), which was set to these configurations: 1) $40 \mathrm{kV}$; 2) $40 \mathrm{~mA}$; 3 ) $\mathrm{CuK} \alpha$ radiation; 4) a scanning rate of $6 \% \mathrm{~min}$; and 5) step size 0.02 , over the $2 \theta$ range of $20^{\circ}-80^{\circ}$. The silver nanoparticles were collected by centrifugation and several washings with sterile water.
Finally, the particles were recovered by air drying the samples and were obtained in powder form.

The stability of silver nanoparticles was observed by keeping the silver nanoparticles in solution at room temperature for different time intervals. In addition, the effect of change in $\mathrm{pH}$ on the stability of the silver nanoparticles was studied. The sodium hydroxide (base) was added in the $\mathrm{pH}$ range of 4-10, and then the solution was scanned by using the UV-Vis spectrophotometer to observe the absorbance.

\section{Analysis of antimicrobial activity of silver nanoparticles}

The antimicrobial activity of the biologically synthesized silver nanoparticles against pathogenic microorganisms B. anthracis, V. parahaemolyticus, S. enterica, E. coli, $B$. cereus. and $C$. albicans was measured on Muller-Hinton agar (MHA) plates by using the well diffusion method. An overnight log culture of each pathogenic strain $(100 \mu \mathrm{L})$ was spread evenly on a MHA plate by using a glass spreader. Wells were made on the MHA plates by using a gel puncture. Then, $50 \mu \mathrm{L}$ of the silver nanoparticle reaction mixture was added into each well, and the wells were incubated at $37^{\circ} \mathrm{C}$ for 24 hours. As a control, $50 \mu \mathrm{L}$ of $1 \mathrm{mM}$ silver nitrate solution was used, this concentration is the same as the one used to biosynthesize the nanoparticles. After incubation, the zones of inhibition were measured by measuring the diameter of the zone formed around each well. Similarly, the experiment was conducted with C. albicans on Sabouraud dextrose agar plates. The study was done in duplicate to check the reproducibility. ${ }^{19}$

Simultaneously, the disc diffusion method was used to investigate the synergistic effect of antibiotics with silver nanoparticles for antimicrobial effect against test strains. In this assay, the MHA medium plates were spread evenly with $100 \mu \mathrm{L}$ of an overnight log culture of test organisms. Each standard antibiotic disc of lincomycin, oleandomycin, vancomycin, novobiocin, penicillin $\mathrm{G}$, and rifampicin was further impregnated with $30 \mu \mathrm{L}(100 \mathrm{mg} / \mathrm{L})$ of freshly prepared, partially purified silver nanoparticle solution and was placed onto the agar plates. For each sample, a corresponding control was maintained by using an antibiotic disc without the silver nanoparticle solution. In addition, a control of only silver nanoparticles and silver nitrate at the same concentrations $(30 \mu \mathrm{L}[100 \mathrm{mg} / \mathrm{L}])$ was maintained. Then, the plates were incubated at $37^{\circ} \mathrm{C}$ for 24 hours. In case of $C$. albicans, cycloheximide $(10 \mu \mathrm{g} / \mathrm{disc})$ was used as a control. After the incubation period, the zones of inhibition around each disc were measured and compared with the corresponding control. ${ }^{20}$ 


\section{Results and discussion}

\section{Screening and identification of bacteria}

After the incubation period, the bacterial strain DC2 showed growth on the TSA plate supplemented with 1 $\mathrm{mM} \mathrm{AgNO}$; this suggests that the bacterial strain DC2 was capable of tolerating silver metal at a $1 \mathrm{mM}$ concentration. On the basis of the molecular characterization of the bacterial isolate, DC2 showed $99.8 \%$ similarity with B. frigoritolerans. B. frigoritolerans is strictly aerobic chemo-organotroph, and it is not as well-known as the other members of the genus Brevibacterium. ${ }^{21}$ Brevibacterium casei has been studied for the biosynthesis of silver and gold nanoparticles. ${ }^{22}$ Biosurfactant-mediated synthesis of silver nanoparticles by marine B. casei MSA19 has been studied. ${ }^{23}$ The reports suggested that the some of the species of the genus do show activity for the biosynthesis of metal nanoparticles; however, to our knowledge, this is the first report of the biosynthesis of silver nanoparticles by $B$. frigoritolerans DC2 isolated from a fermented food product. The 16S rRNA sequence of the strain B. frigoritolerans DC2 has been submitted to National Center for Biotechnology
Information with the accession number KM583447. The strain has been deposited in Korean Collection for Type Culture (KCTC 29680).

\section{Synthesis and characterization of silver nanoparticles}

In the present study, silver nanoparticles were successfully synthesized in the culture supernatant of $B$. frigoritolerans DC2. The formation of silver nanoparticles by the reduction of $\mathrm{AgNO}_{3}$ was indicated by the color change of the reaction mixture (Figure 1A). As the biosynthesis proceeded over 48 hours, the color changed from light yellow to dark brown. This brown color could be due to the excitation of surface plasmon vibrations; if so, this would indicate the formation of silver nanoparticles in the reaction mixture. ${ }^{24}$ On the other hand, no color change was observed in the control flask that contained medium with only the $1 \mathrm{mM}$ $\mathrm{AgNO}_{3}$ solution and had been kept under the same conditions as the other flask (Figure 1B). Because the nanoparticle synthesis was extracellular, the need for downstream processing that would have been otherwise essential for
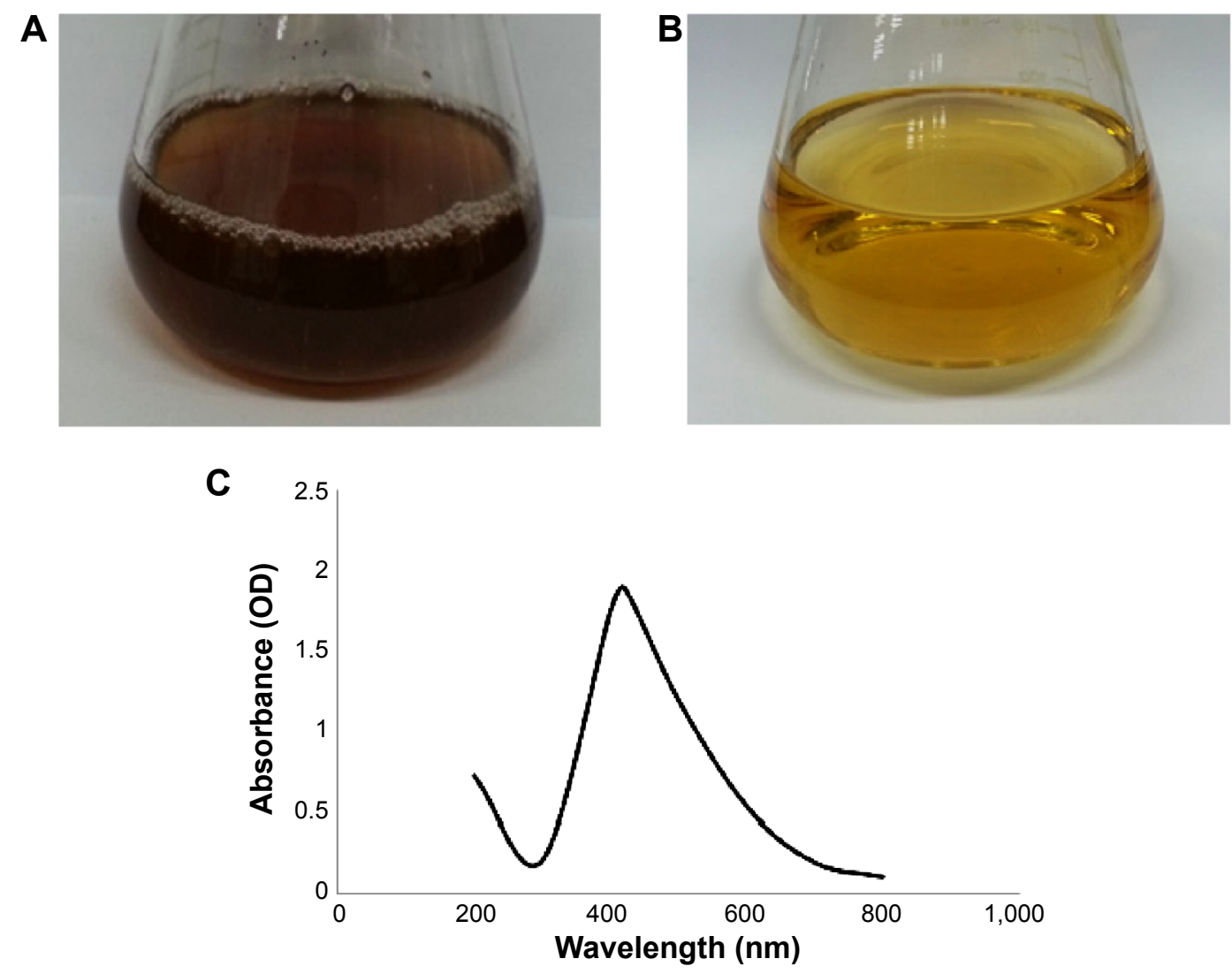

Figure I Chromatic properties of culture supernatant of Brevibacterium frigoritolerans DC2.

Notes: Culture supernatant of $\mathrm{B}$. frigoritolerans $\mathrm{DC} 2$ after incubation with $\mathrm{AgNO}_{3}$ (silver nitrate) $(\mathrm{I} \mathrm{mM})(\mathbf{A})$ and control with medium and $\mathrm{AgNO}{ }_{3}(\mathrm{I} \mathrm{mM})$ after incubation period (B). UV-Vis spectra of culture supernatant of $B$. frigoritolerans DC2 treated with $1 \mathrm{mM} \mathrm{AgNO}_{3}(\mathbf{C})$.

Abbreviations: OD, optical density; UV-Vis, ultraviolet-visible. 
intracellular synthesis was avoided and thereby made the process simpler and more cost-effective. The exact mechanism behind the extracellular synthesis of silver nanoparticles in the supernatant remains to be elucidated. However, reports have suggested that the extracellular enzyme secreted by microorganism in the culture supernatant is responsible for the reduction of silver ions to silver nanoparticles. ${ }^{25,26}$ The study based on extracellular synthesis of silver nanoparticles by Bacillus licheniformis showed that the nitrate reductase enzyme extracellularly secreted by the bacteria in the medium was responsible for the synthesis of silver nanoparticles. ${ }^{8}$

For the characterization of the silver nanoparticles, the reaction mixture was monitored by using UV-Vis spectrophotometer for spectral analysis. After the incubation period, the reaction mixture was scanned in the range of 200-800 nm. In the UV-Vis absorption spectrum, a strong peak at about $420 \mathrm{~nm}$ was observed; this is attributed to the surface plasmon resonance band of the silver nanoparticles (Figure 1C). Reports suggest that the band located in this region corresponds to the surface plasmon resonance of silver nanoparticles. ${ }^{27,28}$ Thus, the reaction mixture indicates the formation of silver nanoparticles.

Various studies have characterized the shape and size of silver nanoparticles by TEM. ${ }^{22,24,27,29}$ In the present study, field emission TEM images of silver nanoparticles revealed that the shape of the nanoparticles was spherical (Figure 2A and $B$ ). The results showed that particles are uniform in shape with $10 \mathrm{~nm}$ to $30 \mathrm{~nm}$ in size. The dynamic light scattering particle size analysis results indicated that the hydrodynamic diameter of the particles range was $50-150 \mathrm{~nm}$ with a 0.191 polydispersity index (Figure 2C-E). The average particle size was $97 \mathrm{~nm} \cdot{ }^{30,31}$ The purity and elemental composition of the biosynthesized silver nanoparticles were determined by EDX, XRD, and elemental mapping. In the EDX spectrum, silver nanocrystallites displayed an optical absorption band peak at approximately $3 \mathrm{keV}$ (Figure 3A); this property is due to the absorption of metallic silver nanocrystallites that correspond to surface plasmon resonance. ${ }^{8}$ Figure $3 \mathrm{~B}$ shows the XRD pattern of silver nanoparticles, which exhibited intense peaks throughout the whole spectrum of $2 \theta$ value, which ranged from $20^{\circ}$ to $80^{\circ}$; this pattern was similar to the Braggs's reflection of silver nanocrystals. Our results are similar to those of previous reports of characterization of silver nanoparticles by XRD. ${ }^{32-35}$

The elemental mapping results of the biosynthesized silver nanoparticles (Figure 3C and D) indicate that silver was distributed maximally $(50.30 \%)$; this finding suggests that silver was the predominant metal. The other elements, such as copper (26.98\%), carbon (14.98\%), and sulfur (1.44\%) were also observed. The distribution of carbon and copper was due to the use of a TEM grid, and slight contamination by chlorine and sulfur appeared. Thus, the results indicate that the strain $B$. frigoritolerans DC2 was found to be capable of synthesizing spherical silver nanoparticles.

The stability of silver nanoparticles was determined by keeping the nanoparticle solution at room temperature for different time intervals over many days. There was no observable variation in the $\mathrm{UV}-\mathrm{V}$ is spectrum of the reaction mixture even after 1 month; this indicated the stable nature of the silver nanoparticles in the reaction mixture. The results proved that the nanoparticles were stable for more than 1 month. In addition, the effect of the change in $\mathrm{pH}$ on the stability of the silver nanoparticles in the $\mathrm{pH}$ range of 4-10 was studied. The silver nanoparticle solution was observed before and after the addition of sodium hydroxide (base). No major change in the wavelength was observed; this result further confirmed the stable nature of the silver nanoparticles.

\section{Antimicrobial activity of silver nanoparticles}

Metal nanoparticles have been shown to be effective against many pathogenic and multidrug-resistant bacteria. For instance, silver nanoparticles have demonstrated activity against methicillin-resistant Staphylococcus aureus (MRSA) and methicillin-resistant Staphylococcus epidermidis (MRSE). ${ }^{36}$ Various studies have reported antimicrobial action of silver nanoparticles against microorganisms. ${ }^{4,37,38}$ In our study, the biosynthesized silver nanoparticles displayed antimicrobial activity against a range of pathogenic microorganisms, such as $C$. albicans, $V$. parahaemolyticus, $S$. enterica, B. anthracis, $B$. cereus, and E. coli.

The antimicrobial activity of the reaction mixture was compared to that of silver nitrate solution after incubation period. Antimicrobial activity against tested microorganisms was measured by measuring the diameter of the zone of inhibition of each well. The results of mean diameters of zones of inhibition are interpreted in Table 1 . The results demonstrate that the reaction mixture containing silver nanoparticles exhibited antimicrobial activity against the tested microorganisms in the following manner, C. albicans followed by V. parahaemolyticus, B. anthracis and B. cereus, $S$. enterica, and then E. coli (Figure 4A-F, respectively). Although the activity of reaction mixture exhibit slightly 
A

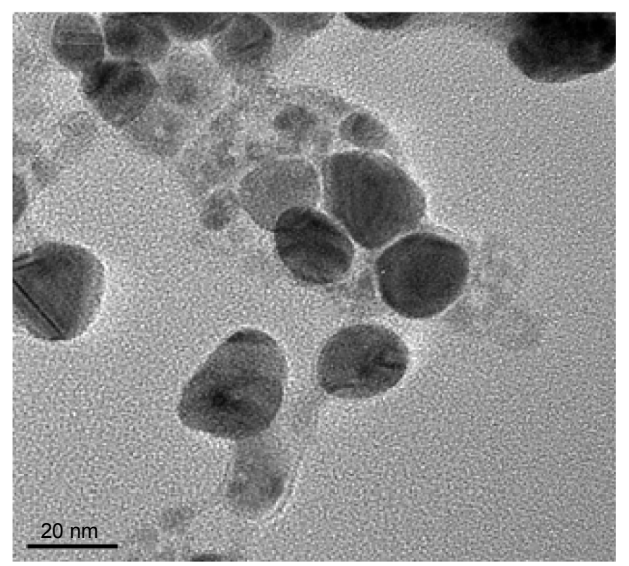

B

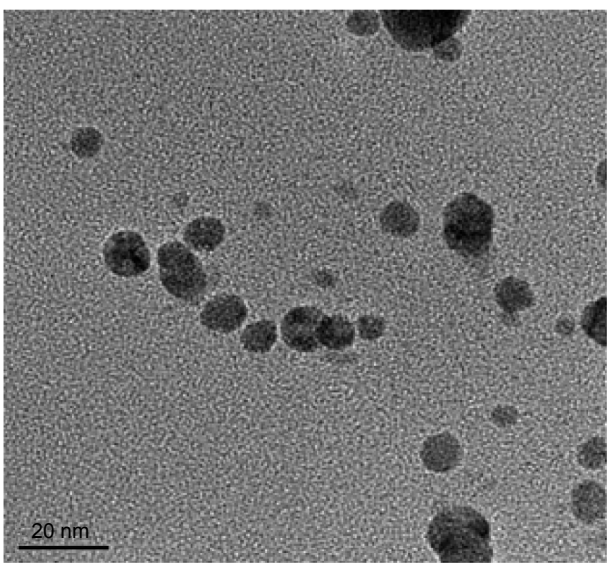

C

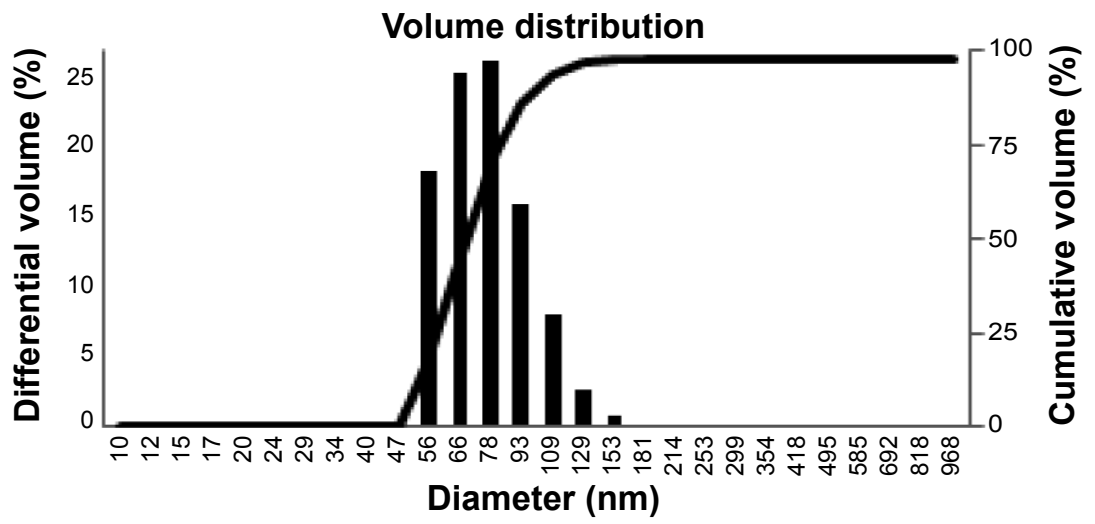

D

Number distribution

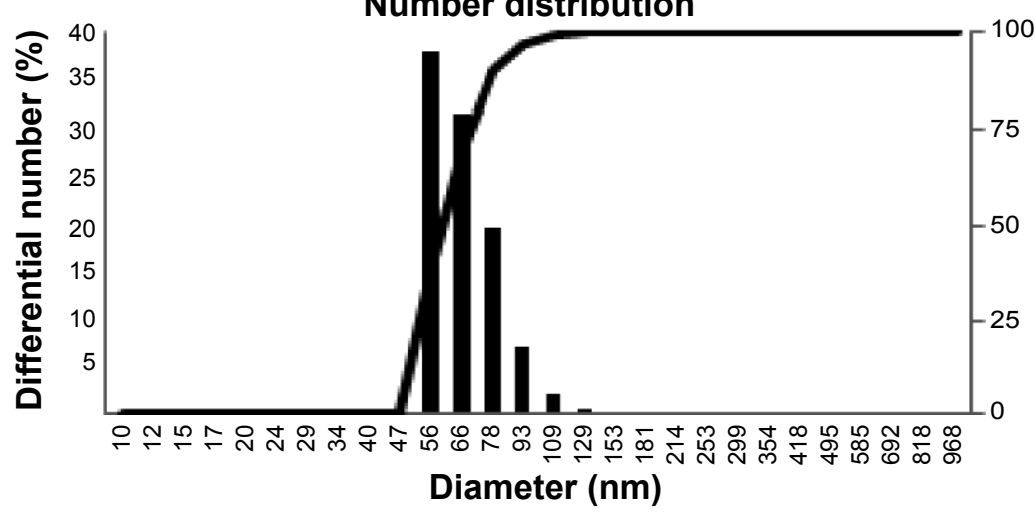

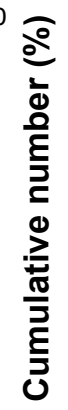

$\mathbf{E}$

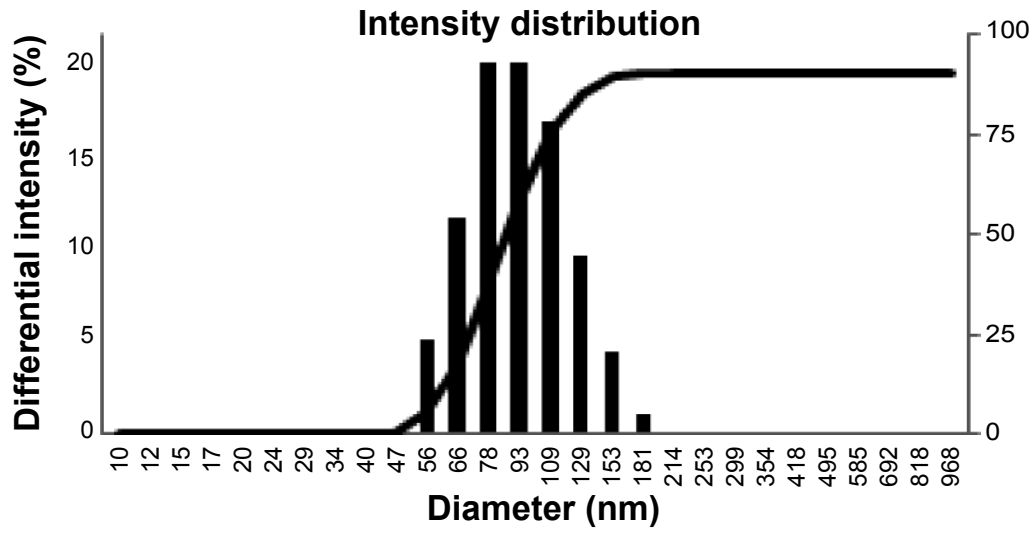

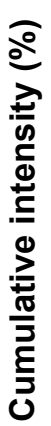

Figure 2 Properties of silver nanoparticles synthesized by Brevibacterium frigoritolerans DC2.

Notes: Transmission electron micrograph of silver nanoparticles synthesized by B. frigoritolerans DC2 (A and B). Particles size distribution of silver nanoparticles according to volume $(\mathbf{C})$, number $(\mathbf{D})$, and intensity $(\mathbf{E})$. 

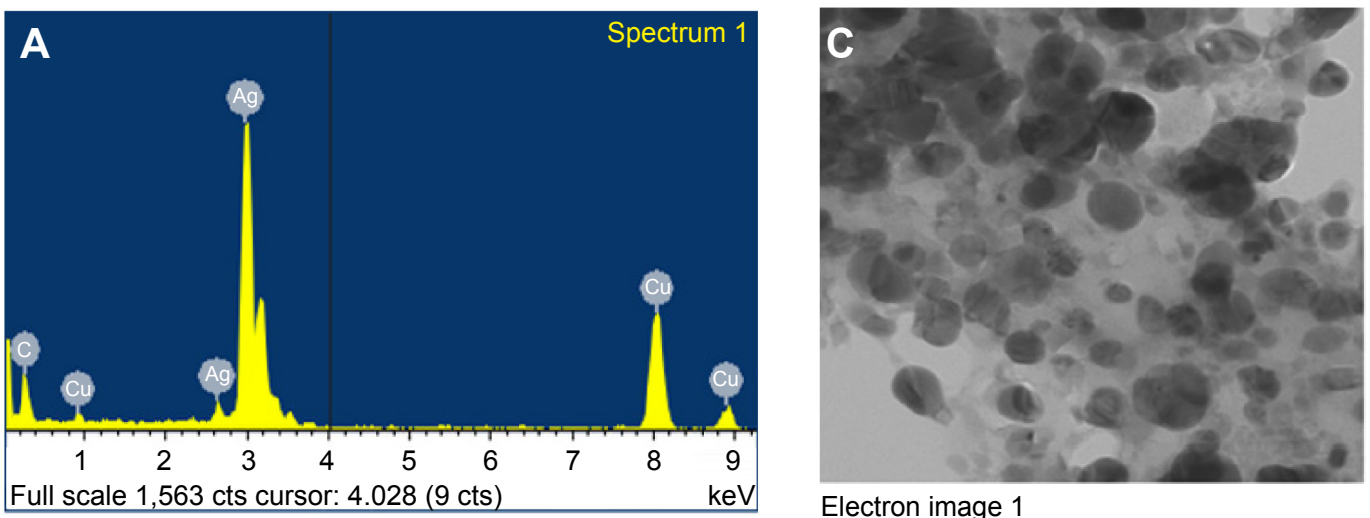

Electron image 1
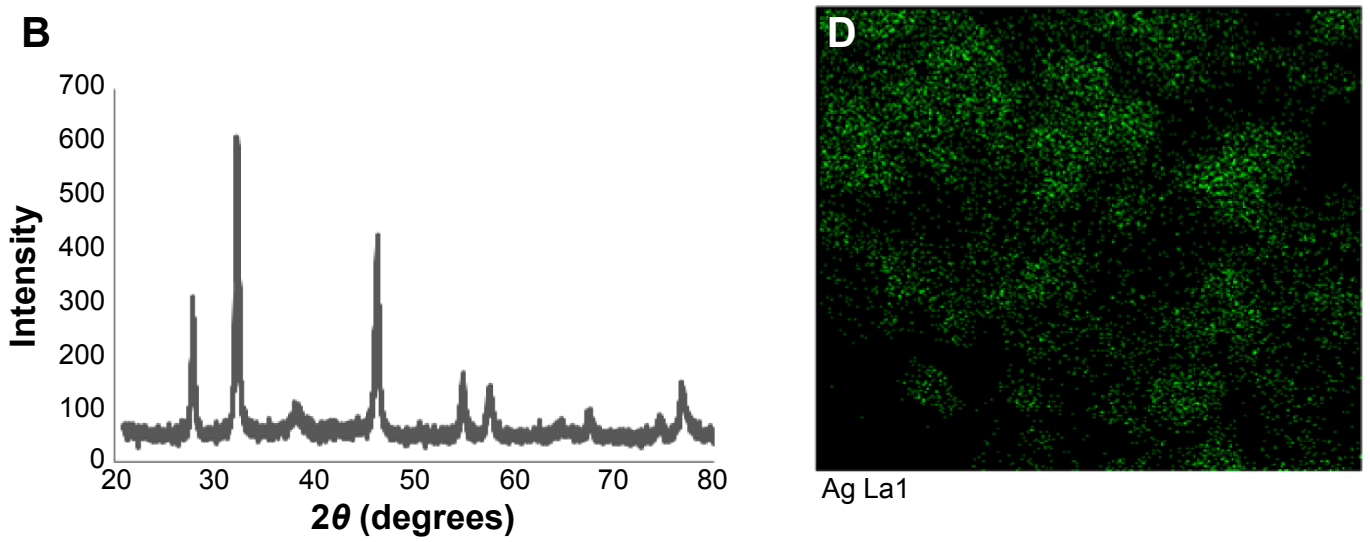

Figure 3 Silver nanoparticles examined under a variety of conditions.

Notes: Energy dispersive X-ray spectroscopy of the whole scan area showing major peak of silver nanoparticles at $3 \mathrm{keV}(\mathbf{A})$; $\mathrm{X}$-ray diffraction patterns of silver nanoparticles obtained by Brevibacterium frigoritolerans DC2 (B); transmission electron micrograph of silver nanoparticle pellet solution (C); silver nanoparticles, green (D).

more antimicrobial activity than silver nitrate solution. The activity was further analyzed after the purification of nanoparticles and compared with that of the same concentration of silver nitrate. The exact mechanism of antimicrobial action is not clearly known, but studies suggest that silver nanoparticles have the ability to damage the cell membrane permeability, damage the respiration functions of the cell,

Table I Diameters of zones of inhibition (mm) for $50 \mu \mathrm{L}$ of reaction mixture containing silver nanoparticles after incubation with various microorganisms

\begin{tabular}{llll}
\hline Number & Microorganism & \multicolumn{2}{l}{$\begin{array}{l}\text { Zone of } \\
\text { inhibition }(\mathbf{m m})\end{array}$} \\
\cline { 3 - 4 } & & $\begin{array}{l}\text { Silver } \\
\text { nitrate }\end{array}$ & $\begin{array}{l}\text { Reaction } \\
\text { mixture }\end{array}$ \\
\hline $\mathrm{I}$ & Candida albicans KACC 30062 & $22 \pm 0.7$ & $24 \pm 1.4$ \\
2 & Vibrio parahaemolyticus ATCC 33844 & $23 \pm \mathrm{I} . \mathrm{I}$ & $25 \pm 1.0$ \\
3 & Bacillus anthracis NCTC 10340 & $14 \pm 0.2$ & $15 \pm 0.8$ \\
4 & Bacillus cereus ATCC I4579 & $14 \pm 0.3$ & $15 \pm 0.5$ \\
5 & Salmonella enterica ATCC I3076 & $12 \pm 0.4$ & $12 \pm 0.6$ \\
6 & Escherichia coli ATCC I0798 & $10 \pm 0.7$ & $1 \mathrm{II} \pm 0.2$ \\
\hline
\end{tabular}

Notes: Each value is the mean of the diameters of two wells. Each well contained $50 \mu \mathrm{L}$ of tested samples. and encourage the formation of free radicals. These factors cause the antimicrobial effect of silver nanoparticles. ${ }^{9,39}$

Several studies have shown that the combination of silver nanoparticles with antibiotics leads to an enhanced effect of the antibiotics against microorganisms. ${ }^{14,20}$ This action is most probably due to an increase in cell wall penetration by these antibiotics with the nanoparticles. Combinations of antibiotics with nanoparticles have many advantages that minimize the side effects of broad-spectrum antibiotics; these benefits include increasing the concentration of the local antibiotic at the target site and facilitating the binding of antibiotics to microorganisms. Moreover, nanoparticleantibiotic conjugates lower the amounts of both the drugs and the nanoparticles in the dosage; this property reduces the side effects of the medication while increasing its antimicrobial properties. ${ }^{14}$ In our study, the synergistic effect of silver nanoparticles with different commercial antibiotics was investigated against six pathogenic microorganisms by using the disc diffusion method. Figure 5A-E represents the antimicrobial activity of partially purified silver nanoparticles and silver nitrate of the same concentration $(1 \mathrm{mM})$ against S. enterica, E. coli, V. parahaemolyticus, B. anthracis, and 

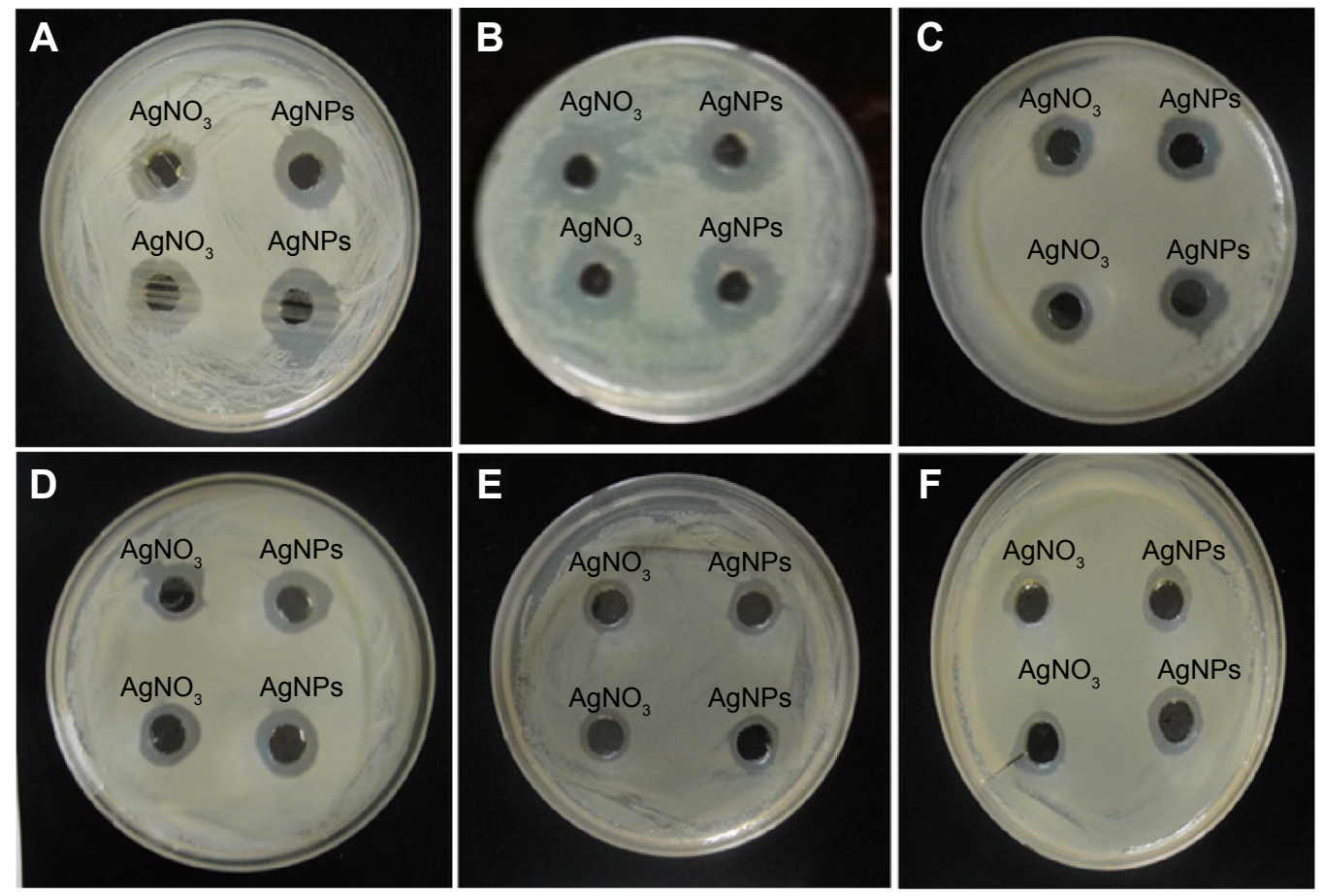

Figure 4 Zones of inhibition for various pathogens.

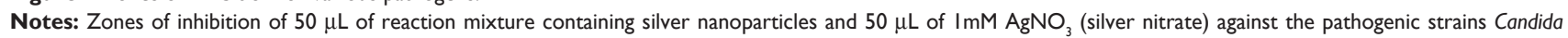
albicans (A), Vibrio parahaemolyticus (B), Bacillus anthracis (C), Bacillus cereus (D), Salmonella enterica (E), and Escherichia coli (F).

Abbreviation: AgNPs, silver nanoparticles.

B. cereus. The appearance of zones of inhibition indicated that the biosynthesized silver nanoparticles had stronger antimicrobial properties against the tested microorganisms than did silver nitrate when both treatments were used at the same concentration. In addition, the synergistic effect of the antibiotics (lincomycin, oleandomycin, novobiocin, vancomycin, penicillin $\mathrm{G}$, and rifampicin) in conjugation with biosynthesized silver nanoparticles increased the sensitivity of the tested microorganisms. The results show that S. enterica, E. coli, and V. parahaemolyticus (Figure 5F-H) were completely resistant to the antibiotics; however, the addition of the silver nanoparticle solutions to the discs rendered the bacterial strains sensitivity to those discs and thereby resulted in the formation of zones of inhibition (Figure $5 \mathrm{~K}-\mathrm{M}$ ). The maximum increase in fold area has been calculated for antibiotics with silver nanoparticles with respect to standard antibiotics. ${ }^{40}$ The average of maximum increase in fold area against the antibiotic-resistant microorganisms S. enterica, E. coli, and V. parahaemolyticus (Figure 6A-C) observed in this study was highest for novobiocin (4.82 fold), then for lincomycin, oleandomycin, and rifampicin (4.23 fold), then for vancomycin (3.98 fold), and finally for penicillin $\mathrm{G}$ (3.66 fold). Various researchers have demonstrated the combined effect of biosynthesized silver nanoparticles with commercial antibiotics. ${ }^{4,14,20}$ The results demonstrate that the silver nanoparticles obtained by B. frigoritolerans DC2 enhance the antimicrobial activity of commercial antibiotics against S. enterica, E. coli, and V. parahaemolyticus.

The other pathogenic strains, B. anthracis and B. cereus (Figure 5I and J), showed a sensitivity pattern to antibiotics. However, when the discs were combined with silver nanoparticles, an increase in the diameters of the zones of inhibition was observed (Figure $5 \mathrm{~N}$ and $\mathrm{O}$ ). The results clearly indicate that the addition of silver nanoparticles enhanced the effect of the antibiotics. Thus, an increase in fold area was observed in all of the antibiotics tested against antibiotic-sensitive and antibiotic-resistant pathogenic microorganisms. Similarly, Figure 5P shows the antimicrobial activity of silver nanoparticles against $C$. albicans. Figure 5Q shows that $C$. albicans was completely resistant to cyclohexamide; however, when the silver nanoparticles were added to the cyclohexamide, antimicrobial activity was enhanced and resulted in the formation of a zone of inhibition. The results showed that the average of maximum increase in fold area against antibiotic-sensitive microorganisms B. anthracis and B. cereus (Figure 6D and E) were, in descending order, as follows: 1) rifampicin (3.69 fold); 2) oleandomycin (1.91 fold); 3) penicillin $G$ (0.36 fold); 4) novobiocin ( 0.26 fold); 5) vancomycin ( 0.22 fold); and 6) lincomycin ( 0.14 fold). Our results are in agreement with 

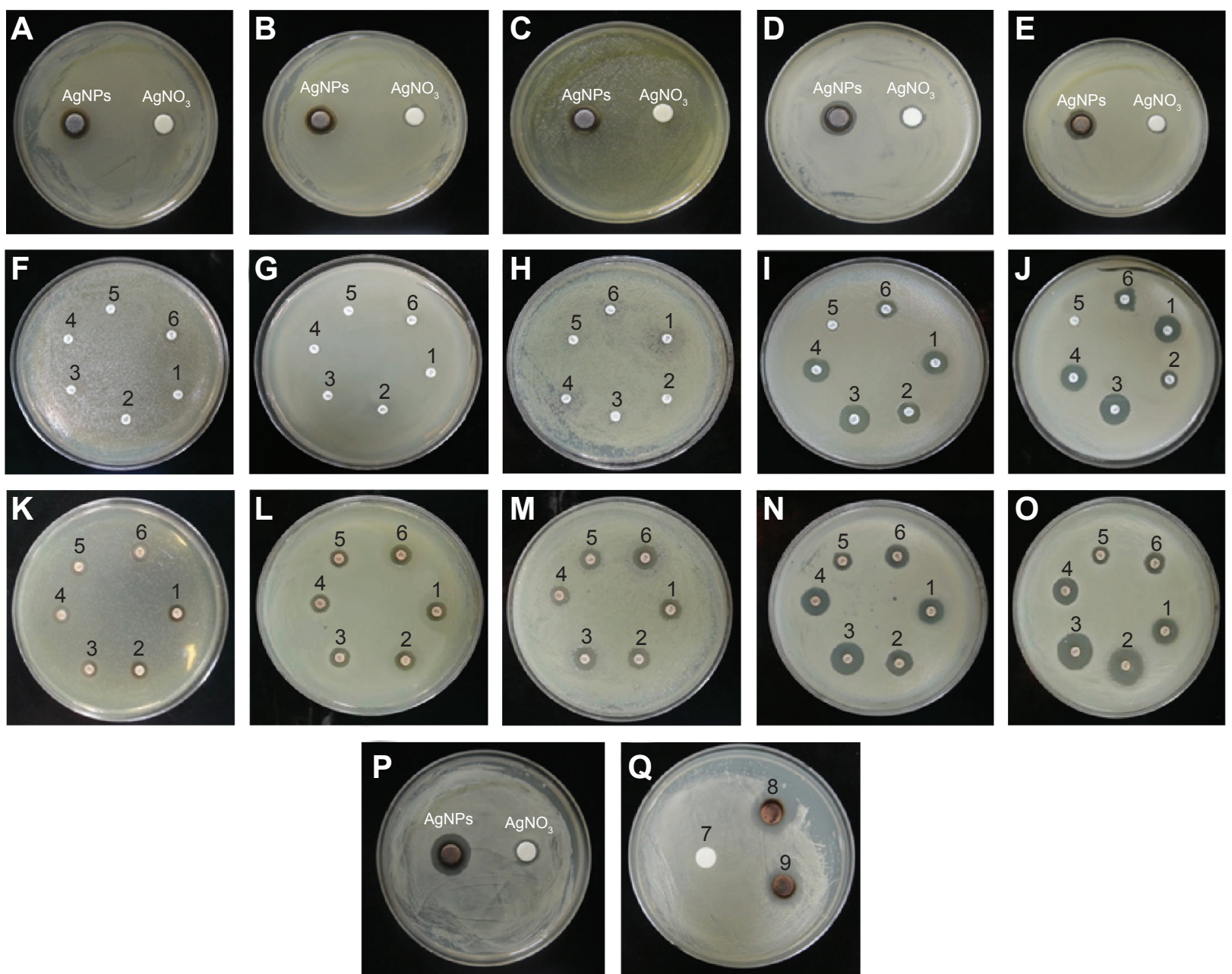

Figure 5 Effects of partially purified silver nanoparticle pellet solution on various pathogens.

Notes: Zones of inhibition of partially purified silver nanoparticle pellet solution and silver nitrate $\left(\mathrm{AgNO}_{3}\right)$ (both at same concentration, $30 \mu \mathrm{L}[\mathrm{I00}$ mg/L]) against Salmonella enterica (A), Escherichia coli (B), Vibrio parahaemolyticus (C), Bacillus anthracis (D), and Bacillus cereus (E). Zones of inhibition of standard antibiotic discs against pathogenic bacteria Salmonella enterica $(\mathbf{F})$, Escherichia coli $(\mathbf{G})$, Vibrio parahaemolyticus $(\mathbf{H})$, Bacillus anthracis $(\mathbf{I})$, and Bacillus cereus $(\mathbf{J})$. Zones of inhibition of standard antibiotic discs with

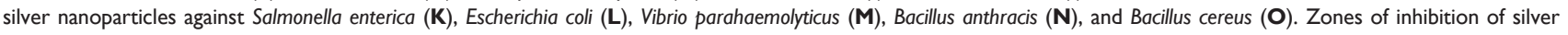
nanoparticles and silver nitrate against Candida albicans ( $\mathbf{P})$, cycloheximide (Q-7), Cycloheximide with AgNPs (Q-8,9). For (A-Q), the numbers, the corresponding antibiotics, and the corresponding concentrations of antibiotics are as follows: I) lincomycin (MYI5), $15 \mu \mathrm{g} /$ disc; 2) oleandomycin (OLI5), I5 $\mu \mathrm{g} / \mathrm{disc} ; 3)$ novobiocin (NV30), $30 \mu \mathrm{g} /$ disc; 4) vancomycin (VA30), $30 \mu \mathrm{g} /$ disc; 5) penicillin G (PI0), $10 \mu \mathrm{g} /$ disc; 6) rifampicin (RD5) $5 \mu \mathrm{g} /$ disc; 7) cycloheximide, $10 \mu \mathrm{g} / \mathrm{disc}$; 8 cycloheximide (I0 $\mu \mathrm{g} /$ disc) with silver nanoparticles; and 9) cycloheximide ( $10 \mu \mathrm{g} /$ disc) with silver nanoparticles.

Abbreviation: AgNPs, silver nanoparticles.

other studies that have shown the antimicrobial activity of silver nanoparticles against many pathogenic and multidrugresistant microorganisms. ${ }^{4,14}$ In our findings, biosynthesized silver nanoparticles showed antimicrobial activity against pathogenic microorganisms. Moreover, the silver nanoparticles enhanced the antimicrobial activity of commercial antibiotics against pathogenic microorganisms. However, the exact action mechanism still must be explored.

\section{Conclusion}

In our study, the method for biological synthesis of silver nanoparticles by $B$. frigoritolerans DC2 was established. This biogenic process will be helpful for minimizing the need for chemical and physical methodologies. In addition to this, the extracellular synthesis of nanoparticles could be highly advantageous for facilitating downstream processing in large-scale operations. The silver nanoparticles that were obtained showed antimicrobial effects against various pathogenic microorganisms. Furthermore, they enhanced the antimicrobial activity of various commercial antibiotics against pathogenic microorganisms.

\section{Acknowledgments}

This research was supported by the program of Agriculture Science \& Technology Development (grant\#: PJ008813042014), Rural Development Administration and also supported by a grant from the Next-Generation BioGreen 21 Program (SSAC, grant\#: PJ00952903). 


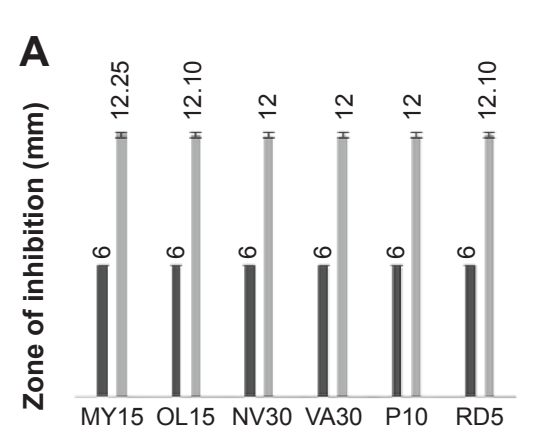

Antibiotic

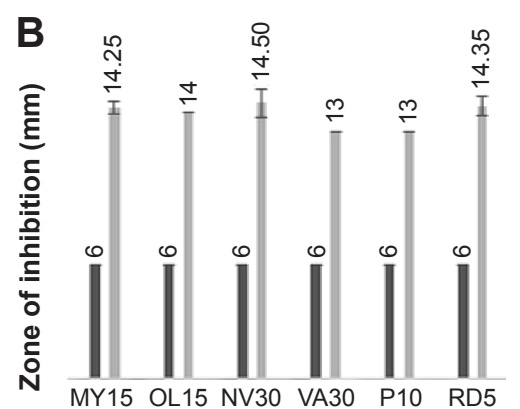

Antibiotic

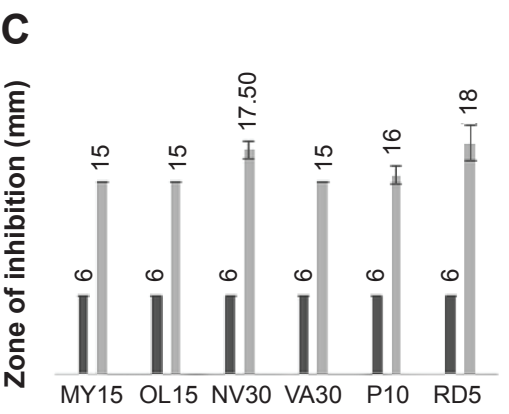

Antibiotic

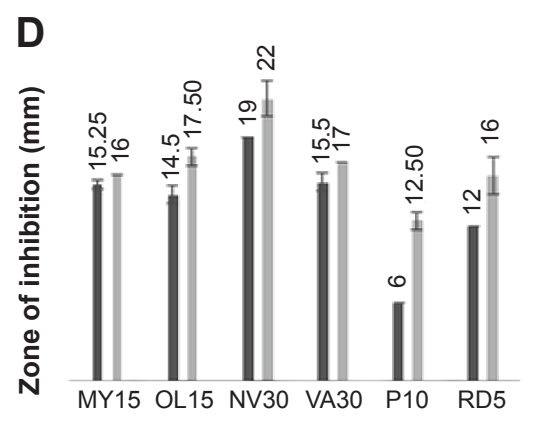

Antibiotic

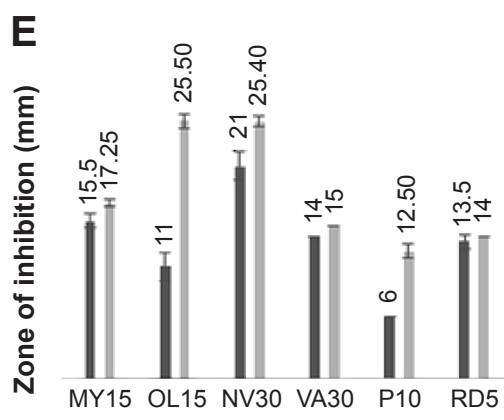

Antibiotic

Standard antibiotic Standard antibiotic with silver nanoparticles $(30 \mu \mathrm{L},[100 \mathrm{mg} / \mathrm{L}])$

Figure 6 Graphical representation of comparative study between antibiotics and antibiotics with AgNPs.

Notes: Salmonella enterica (A), Escherichia coli (B), Vibrio parahaemolyticus (C), Bacillus anthracis (D), and Bacillus cereus (E), respectively. Results interpreting the antimicrobial activity of commercial antibiotics alone with that of antibiotics combined with silver nanoparticles.

\section{Disclosure}

The authors report no conflicts of interest in this work.

\section{References}

1. Shankar SS, Rai A, Ahmad A, Sastry M. Rapid synthesis of Au, Ag, and bimetallic Au core-Ag shell nanoparticles using Neem (Azadirachta indica) leaf broth. J Colloid Interf Sci. 2004;275(2):496-502.

2. Raut RW, Lakkakula JR, Kolekar NS, Mendhulkar VD, Kashid SB. Phytosynthesis of silver nanoparticle using Gliricidia sepium (Jacq). Curr Nanosci. 2009;5:117-122.

3. Nadagouda MN, Varma RS. Green synthesis of silver and palladium nanoparticles at room temperature using coffee and tea extract. Green Chem. 2008;10(8):859-862.

4. Dar MA, Ingle A, Rai M. Enhanced antimicrobial activity of silver nanoparticles synthesized by Cryphonectria sp. evaluated singly and in combination with antibiotics. Nanomedicine. 2013;9(1):105-110.

5. Kumar V, Yadav SK. Plant-mediated synthesis of silver and gold nanoparticles and their applications. JChem Technol Biotechnol. 2009;84(2): 151-157.

6. Ahmad A, Senapati S, Khan MI, et al. Intracellular synthesis of gold nanoparticles by a novel alkalotolerant actinomycete, Rhodococcus species. Nanotechnology. 2003;14:824-828.

7. Basavaraja S, Balaji SD, Lagashetty A, Rajasab AH, Venkataraman A. Extracellular biosynthesis of silver nanoparticles using the fungus Fusarium semitectum. Mater Res Bull. 2008;43(5):1164-1170.

8. Kalimuthu K, Babu RS, Venkataraman D, Bilal M, Gurunathan S. Biosynthesis of silver nanocrystals by Bacillus licheniformis. Colloids Surf B Biointerfaces. 2008;65(1):150-153.

9. Prabhu S, Poulose EK. Silver nanoparticles: mechanism of antimicrobial action, synthesis, medical applications, and toxicity effects. International Nano Letters. 2012;2(1):1-10.
10. Sibanda T, Okoh AI. The challenges of overcoming antibiotic resistance: Plant extracts as potential sources of antimicrobial and resistance modifying agents. African Journal of Biotechnology. 2007;6(25):2886-2896.

11. Kollef MH, Golan Y, Micek ST, Shorr AF, Restrepo MI. Appraising contemporary strategies to combat multidrug resistant gram-negative bacterial infections-proceedings and data from the Gram-Negative Resistance Summit. Clin Infect Dis. 2011;53(Suppl 2):S33-S55; quiz S56-S58.

12. Rai MK, Deshmukh SD, Ingle AP, Gade AK. Silver nanoparticles: the powerful nanoweapon against multidrug-resistant bacteria. $J$ Appl Microbiol. 2012;112(5):841-852.

13. Rosarin FS, Mirunalini S. Nobel metallic nanoparticles with novel biomedical properties. J Bioanal Biomed. 2011;3:085-091.

14. Fayaz AM, Balaji K, Girilal M, Yadav R, Kalaichelvan PT, Venketesan R. Biogenic synthesis of silver nanoparticles and their synergistic effect with antibiotics: a study against gram-positive and gram-negative bacteria. Nanomedicine. 2010;6(1):103-109.

15. Weisburg WG, Barns SM, Pelletier DA, Lane DJ. 16S ribosomal DNA amplification for phylogenetic study. J Bacteriol. 1991;173(2): 697-703.

16. Kim KK, Kim MK, Lim JH, Park HY, Lee ST. Transfer of Chryseobacterium meningosepticum and Chryseobacterium miricola to Elizabethkingia gen. nov. as Elizabethkingia meningoseptica comb. nov. and Elizabethkingia miricola comb. nov. Int J Syst Evol Microbiol. 2005;55(Pt 3):1287-1293.

17. Kim OS, Cho YJ, Lee K, et al. Introducing EzTaxon-e: a prokaryotic $16 \mathrm{~S}$ rRNA gene sequence database with phylotypes that represent uncultured species. Int J Syst Evol Microbiol. 2012;62(Pt 3):716-721.

18. Supraja S, Mohammed Ali S, Chakravarthy N, et al. Green synthesis of silver nanoparticles from Cynodon dactylon leaf extract. Int $J$ Chem Tech. 2013;5(1):271-277. 
19. Logeswari P, Silambarasan S, Abraham J. Ecofriendly synthesis of silver nanoparticles from commercially available plant powders and their antibacterial properties. Scientia Iranica. 2013;20(3):1049-1054.

20. Naqvi SZH, Kiran U, Ali MI, et al. Combined efficacy of biologically synthesized silver nanoparticles and different antibiotics against multidrug-resistant bacteria. Int J Nanomedicine. 2013;8:3187-3195.

21. Selvakumar G, Sushil SN, Stanley J, et al. Brevibacterium frigoritolerans a novel entomopathogen of Anomala dimidiata and Holotrichia longipennis (Scarabaeidae: Coleoptera). Biocontrol Science and Technology. 2011;21(7):821-827.

22. Kalishwaralal K, Deepak V, Pandian SRK, et al. Biosynthesis of silver and gold nanoparticles using Brevibacterium casei. Colloids Surf B Biointerfaces. 2010;77:257-262.

23. Kiran GS, Sabu A, Selvin J. Synthesis of silver nanoparticles by glycolipid biosurfactant produced from marine Brevibacterium casei MSA19. J Biotechnol. 2010;148(4):221-225.

24. Balaji DS, Basavaraja S, Deshpande R, Mahesh D, Prabhakar BK, Venkataraman A. Extracellular biosynthesis of functionalized silver nanoparticles by strains of Cladosporium cladosporioides fungus. Colloids Surf B Biointerfaces. 2009;68(1):88-92.

25. Das VL, Thomas R, Varghese RT, Soniya EV, Mathew J, Radhakrishnan EK. Extracellular synthesis of silver nanoparticles by the Bacillus strain CS 11 isolated from industrialized area. 3 Biotech. 2014;4(2): 121-126.

26. Durán N, Marcato PD, Alves OL, De Souza G, Esposito E. Mechanistic aspects of biosynthesis of silver nanoparticles by several Fusarium oxysporum strains. J Nanobiotechnol. 2005;3(8):1-7.

27. Vidhu VK, Aromal S, Philip D. Green synthesis of silver nanoparticles using Macrotyloma uniflorum. Spectrochimica Acta Part A: Molecular and Biomolecular Spectroscopy. 2011;83(1):392-397.

28. Wani IA, Khatoon S, Ganguly A, Ahmed J, Ganguli AK, Ahmad T. Silver nanoparticles: Large scale solvothermal synthesis and optical properties. Mater Res Bull. 2010;45(8):1033-1038.

29. Litvin VA, Minaev BF. Spectroscopy study of silver nanoparticles fabrication using synthetic humic substances and their antimicrobial activity. Spectrochim. Acta, Part A. 2013;108:115-122.
30. Vithiya K, Kumar R, Sen S. Bacillus sp. mediated extracellular synthesis of silver nanoparticles. International Journal of Pharmcy and Pharmaceutical Sciences. 2014;6(Suppl 2):525-527.

31. Otari SV, Patil RM, Nadaf NH, Ghosh SJ, Pawar SH. Green biosynthesis of silver nanoparticles from an actinobacteria Rhodococcus sp. Mater Lett. 2012;72:92-94.

32. Litvin VA, Galagan RL, Minaev BF. Kinetic and mechanism formation of silver nanoparticles coated by synthetic humic substances. Colloids Surf A Physicochem Eng Asp. 2012;414:234-243.

33. Wani IA, Ganguly A, Ahmed J, Ahmad T. Silver nanoparticles: Ultrasonic wave assisted synthesis, optical characterization and surface area studies. Mater Lett. 2011;65(3):520-522.

34. Nabikhan A, Kandasamy K, Raj A, Alikunhi NM. Synthesis of antimicrobial silver nanoparticles by callus and leaf extracts from saltmarsh plant, Sesuvium portulacastrum. Colloids Surf B Biointerfaces. 2010; 79(2):488-493.

35. Gnanadesigan M, Anand M, Ravikumar S, et al. Antibacterial potential of biosynthesised silver nanoparticles using Avicennia marina mangrove plant. Appl Nanosci. 2012;2(2):143-147.

36. Saravanan M, Nanda A. Extracellular synthesis of silver bionanoparticles from Aspergillus clavatus and its antimicrobial activity against MRSA and MRSE. Colloids Surf B Biointerfaces. 2010;77(2):214-218.

37. Ahmad T, Wani IA, Manzoor N, Ahmed J, Asiri AM. Biosynthesis, structural characterization and antimicrobial activity of gold and silver nanoparticles. Colloids Surf B Biointerfaces. 2013;107:227-234.

38. Wani IA, Khatoon S, Ganguly A, Ahmed J, Ahmad T, Manzoor N Structural characterization and antimicrobial properties of silver nanoparticles prepared by inverse microemulsion method. Colloids Surf B Biointerfaces. 2013;101:243-250.

39. Lemire JA, Harrison JJ, Turner RJ. Antimicrobial activity of metals: mechanisms, molecular targets and applications. Nat Rev Microbiol. 2013;11(6):371-384.

40. Birla SS, Tiwari VV, Gade AK, Ingle AP, Yadav AP, Rai MK. Fabrication of silver nanoparticles by Phoma glomerata and its combined effect against Escherichia coli, Pseudomonas aeruginosa and Staphylococcus aureus. Lett Appl Microbiol. 2009;48(2):173-179.
International Journal of Nanomedicine

\section{Publish your work in this journal}

The International Journal of Nanomedicine is an international, peerreviewed journal focusing on the application of nanotechnology in diagnostics, therapeutics, and drug delivery systems throughout the biomedical field. This journal is indexed on PubMed Central,

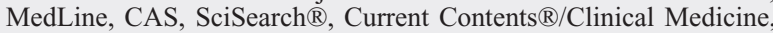

\section{Dovepress}

Journal Citation Reports/Science Edition, EMBase, Scopus and the Elsevier Bibliographic databases. The manuscript management system is completely online and includes a very quick and fair peer-review system, which is all easy to use. Visit http://www.dovepress.com/ testimonials.php to read real quotes from published authors. 